\title{
55. On Families of Effective Divisors on Algebraic Manifolds
}

\author{
By Makoto NAMBA*) \\ Tohoku University \\ (Communicated by Kunihiko KodAIRA, M. J. A., Nov. 12, 1977)
}

1. By an algebraic manifold, we mean a connected compact complex manifold imbedded in a complex projective space. Let $V$ be an algebraic manifold. We denote by $\operatorname{Pic}^{0}(V)$ the set of all holomorphic line bundles on $V$ whose Chern classes vanish. It is well known that $\operatorname{Pic}^{0}(V)$ is an abelian variety of dimension $q=\operatorname{dim} H^{1}(V, \mathcal{O})$, the irregularity of $V$. Let $c \in H^{2}(\mathrm{~V}, Z)$ be a cohomology class of type $(1,1)$. We put

$$
D^{c}(V)=\{D \mid D \text { is an effective divisor on } V \text { with } c([D])=c\},
$$

where $[D]$ is the line bundle determined by $D$ and $c([D])$ is the Chern class of $[D]$. According to Weil [7] (see also Kodaira [3]), $D^{c}(V)$ is a projective variety (i.e., a complex space imbedded in a complex projective space) and the Jacobi mapping

$$
\Phi: D \in D^{c}(V) \rightarrow\left[D-D_{o}\right] \in \operatorname{Pic}^{0}(V)
$$

is holomorphic, where $D_{o} \in \boldsymbol{D}^{c}(V)$ is a fixed effective divisor.

In this note, we state the following theorems. Details will be published elsewhere.

Theorem 1. Assume that there is an effective divisor $D \in D^{c}(V)$ such that

$$
\operatorname{dim} H^{0}(V, \mathcal{O}([D]))>\operatorname{dim} H^{1}(V, \mathcal{O}([D])) .
$$

Then the Jacobi mapping $\Phi$ is surjective and each fiber of $\Phi$ has dimension at least $\operatorname{dim} H^{0}(V, \mathcal{O}([D]))-\operatorname{dim} H^{1}(V, \mathcal{O}([D]))-1$.

In general, we put $W_{c}=\Phi\left(\boldsymbol{D}^{c}(V)\right)$. It is a closed subvariety of $\operatorname{Pic}^{0}(V)$.

Theorem 2. For an effective divisor $D \in D^{c}(V)$, put $a=$ $\operatorname{dim} H^{\circ}(V, \mathcal{O}([D]))$ and $b=\operatorname{dim} H^{1}(V, \mathcal{O}([D]))$. Assume that $a \leqq b$. Then there are an open neighbourhood $U$ of $x=\Phi(D)$ in $\mathrm{Pic}^{0}(V)$ and $a$ $(a \times b)$-matrix valued holomorphic function $A(y), y \in U$, on $U$ such that $W_{c} \cap U$ is the set of zeros of all $a \times a$ minors of $A(y)$.

Remark. If $V$ is a non-singular curve, then Theorem 1 reduces to Jacobi inversion problem and Theorem 2 reduces to Kempf's theorem [5].

2. Theorem 1 and Theorem 2 are easy consequences of the fol-

*) Supported by Alexander von Humboldt Foundation. 
lowing theorems. In the sequel, by a complex space, we mean a reduced, Hausdorff, complex analytic space.

Theorem 3. Let $\left\{V_{s}\right\}_{s \in S}$ be a family of compact complex manifolds with the parameter space $S$, a complex space. Let $\left\{F_{s}\right\}_{s \in S}$ be a family of holomorphic vector bundles over $\left\{V_{s}\right\}_{s \in S}$. Then, for each point $o \in S$, there are an open neighbourhood $S^{\prime}$ of o in $S$ and a vector bundle homomorphism

$$
u: H^{0}\left(V_{o}, \mathcal{O}\left(F_{o}\right)\right) \times S^{\prime} \rightarrow H^{1}\left(V_{o}, \mathcal{O}\left(F_{o}\right)\right) \times S^{\prime}
$$

such that the union $\bigcup_{s \in S^{\prime}} H^{\circ}\left(V_{s}, \mathcal{O}\left(F_{s}\right)\right)$ is identified with the kernel of $u$.

The following Theorem 4 and Theorem 5 are considered as special cases of Schuster [6].

Theorem 4. Let $\left\{V_{s}\right\}_{s \in S}$ and $\left\{F_{s}\right\}_{s \in S}$ be as in Theorem 3. Then the union $\boldsymbol{H}=\bigcup_{s \in S} H^{0}\left(V_{s}, \mathcal{O}\left(F_{s}\right)\right)$ admits a complex space structure so that $(\boldsymbol{H}, \lambda, S)$ is a complex linear space in the sense of Grauert [1], where $\lambda: H \rightarrow S$ is the canonical projection.

Theorem 5. Let $\left\{V_{s}\right\}_{s \in S}$ and $\left\{F_{s}\right\}_{s \in S}$ be as in Theorem 3. Let $P\left(F_{s}\right)$ be the projective space associated with $H^{0}\left(V_{s}, \mathcal{O}\left(F_{s}\right)\right) . \quad\left(P\left(F_{s}\right)\right.$ is empty if $H^{0}\left(V_{s}, \mathcal{O}\left(F_{s}\right)\right)=0$.) Then the union $\boldsymbol{P}=\bigcup_{s \in S} P\left(F_{s}\right)$ admits a complex space structure so that the canonical projection $\mu: P \rightarrow S$ is a proper holomorphic mapping.

By Theorem 3, we easily get

Theorem 6. Let $\left\{V_{s}\right\}_{s \in S},\left\{\boldsymbol{F}_{s}\right\}_{s \in S}$ and $\mu: P \rightarrow S$ be as in Theorem 5 . For a point $o \in S$, assume that $\operatorname{dim} H^{0}\left(V_{o}, \mathcal{O}\left(F_{o}\right)\right)>\operatorname{dim} H^{1}\left(V_{o}, \mathcal{O}\left(F_{o}\right)\right)$. Then there is an open neighbourhood $S^{\prime}$ of $o$ in $S$ such that $\mu^{\prime}$ $=\mu \mid \mu^{-1}\left(S^{\prime}\right): \mu^{-1}\left(S^{\prime}\right) \rightarrow S^{\prime}$ is surjective and each fiber of $\mu^{\prime}$ has dimension at least $\operatorname{dim} H^{0}\left(V_{o}, \mathcal{O}\left(F_{o}\right)\right)-\operatorname{dim} H^{1}\left(V_{o}, \mathcal{O}\left(F_{o}\right)\right)-1$.

Theorem 7. Let $\left\{V_{s}\right\}_{s \in S},\left\{F_{s}\right\}_{s \in S}$ and $\mu: P \rightarrow S$ be as in Theorem 5 . For a point $o \in S$, put $a=\operatorname{dim} H^{0}\left(V_{o}, \mathcal{O}\left(\boldsymbol{F}_{o}\right)\right)$ and $b=\operatorname{dim} H^{1}\left(V_{o}, \mathcal{O}\left(\boldsymbol{F}_{o}\right)\right)$. Assume that $a \leqq b$. Then there are an open neighbourhood $S^{\prime}$ of o in $S$ and $a(a \times b)$-matrix valued holomorphic function $A(s), s \in S^{\prime}$, on $S^{\prime}$ such that $\mu(\boldsymbol{P}) \cap S^{\prime}$ is the set of zeros of all $a \times a$ minors of $A(s)$.

3. In order to get Theorem 1 and Theorem 2 from the theorems in $\S 2$, we consider the case

$$
\begin{gathered}
S=\operatorname{Pic}^{0}(V), \\
V_{x}=V(\text { fixed), } \\
F_{x}=B_{x} \otimes\left[D_{o}\right],
\end{gathered}
$$

where $B_{x}$ is the line bundle, with the Chern class 0 , corresponding to the point $x \in \mathrm{Pic}^{0}(V)$. Then we can easily prove that the complex space $\boldsymbol{P}$ in Theorem 5 is canonically biholomorphic to $\boldsymbol{D}^{c}(V)$. Now, Theorem 6 and Theorem 7 reduce to Theorem 1 and Theorem 2, respectively. 
4. Let $x \in \mathrm{Pic}^{0}(V)$. Let

$$
\sigma: H^{0}\left(V, \mathcal{O}\left(F_{x}\right)\right) \times H^{1}(V, \mathcal{O}) \rightarrow H^{1}\left(V, \mathcal{O}\left(F_{x}\right)\right)
$$

be the bilinear map defined by

$$
\sigma(\xi, h)_{i k}(z)=\xi_{i}(z) h_{i k}(z),
$$

where $\xi=\left\{\xi_{i}(z)\right\} \in H^{0}\left(V, \mathcal{O}\left(F_{x}\right)\right)$ and $h=\left\{h_{i k}(z)\right\} \in H^{1}(V, \mathcal{O})$ for a suitable Stein open covering $\left\{U_{i}\right\}$ of $V$. We put

$$
\sigma(\xi, h)=\sigma_{\xi}(h)=\sigma(h)(\xi)
$$

by abuse of notation. Let $D=(\xi)$ be the zero divisor of $\xi$. $D$ is said to be semi-regular if and only if the linear map $\sigma_{\xi}: H^{1}(V, \mathcal{O})$ $\rightarrow H^{1}\left(V, \mathcal{O}\left(F_{x}\right)\right)$ is surjective. Note that if $V$ is a non-singular curve, then every $D$ is semi-regular.

The semi-regularity theorem by Kodaira-Spencer [4] says that if $D$ is semi-regular, then $D$ is a non-singular point of $D^{c}(V)$ and

$$
\operatorname{dim}_{D} D^{c}(V)=\operatorname{dim} H^{0}(V, \mathcal{O}([D]))-\operatorname{dim} H^{1}(V, \mathcal{O}([D]))+q-1 .
$$

We note that the differential at $(\xi, x)$ of the mapping $u$ in Theorem 3 is equal to

$$
\left(\begin{array}{cc}
0 & \sigma_{\xi} \\
0 & 1
\end{array}\right)
$$

in our case. From this fact, we get the semi-regularity theorem.

Finally, we generalize Kempf's theorems [2] as follows: For a point $x \in \mathrm{Pic}^{0}(V)$, assume that every divisor in $\Phi^{-1}(x)$ is semi-regular. Let $N$ and $C$ be the normal bundle of $D^{c}(V)$ along $\Phi^{-1}(x)$ and the tangent cone of $W_{c}=\Phi\left(D^{c}(V)\right)$ at $x$, respectively. Let

be the mapping induce by $\Phi$.

$$
\rho: N \rightarrow C
$$

Kempf's theorem for algebraic manifolds (c.f. Kempf [2]). For a point $x \in \operatorname{Pic}^{0}(V)$, assume that every divisor in $\Phi^{-1}(x)$ is semi-regular. Assume moreover that

Then

$$
\operatorname{dim} H^{0}\left(V, \mathcal{O}\left(F_{x}\right)\right) \leqq \operatorname{dim} H^{1}\left(V, \mathcal{O}\left(F_{x}\right)\right)+1 .
$$

(1) $\rho: N \rightarrow C$ is a rational resolution.

(2) The degree of $C$ is the binomial coefficient

$$
\left(\begin{array}{l}
\operatorname{dim} H^{1}\left(V, \mathcal{O}\left(F_{x}\right)\right) \\
\operatorname{dim} H^{0}\left(V, \mathcal{O}\left(F_{x}\right)\right)-1
\end{array}\right) .
$$

(3) If $\operatorname{dim} H^{0}\left(V, \mathcal{O}\left(F_{x}\right)\right) \leqq \operatorname{dim} H^{1}\left(V, \mathcal{O}\left(F_{x}\right)\right)$, then the ideal defining $C$ is generated by the maximal minors of the matrix valued function $\sigma(h)$ on $H^{1}(V, \mathcal{O})$.

\section{References}

[1] H. Grauert: Über Modifikationen und exzeptionelle analytische Mengen. Math. Ann., 146, 331-368 (1962). 
[2] J. Kempf: On the geometry of a theorem of Riemann. Ann. Math., 98, 178-185 (1973).

[3] K. Kodaira: Characteristic linear systems of complete continuous systems. Amer. J. Math., 78, 716-744 (1956).

[4] K. Kodaira and D. C. Spencer: A theorem of completeness of characteristic systems of complete continuous systems. Amer. J. Math., 81, 477-500 (1959).

[5] D. Mumford: Curves and their Jacobians. The University of Michigan Press, 1975.

[6] H. Schuster: Zur Theorie der Deformationen kompakter komplexer Räume. Inv. Math., 9, 284-294 (1970).

[7] A. Weil: On Picard varieties. Amer. J. Math., 74, 865-894 (1952). 
